\title{
Feasibility of Hybrid RANS-LES Modeling of Shock/Boundary-Layer Interaction in a Duct
}

\author{
S. Arvidson, S-H. Peng and L. Davidson
}

\begin{abstract}
A shock induced boundary-layer separation (SBLI) occurring in a duct at $M=1.4$ has been analyzed using hybrid RANS-LES methods. The shock wave interacts with the turbulent wall boundary layers and triggers flow separation in the duct corners. The main purpose of the present work is to highlight the difficulties in modeling SBLI, particularly, when hybrid RANS-LES models are used. Results computed using different turbulence models are presented and discussed in comparison with available experimental data. Based on a number of simulations, some issues are addressed and some critical remarks are provided for potential improvements using turbulence-resolving modeling approaches in future work.
\end{abstract}

\section{Introduction}

Shock/boundary-layer interaction (SBLI) is a common flow phenomenon that requires special attention in designing aerial vehicles at trans- and supersonic speeds. Flows passing propelling nozzles, air inlets and wings at transonic and supersonic speeds are, among others, typical examples where shock/boundary-layer interaction may occur. SBLI often leads to extensive pressure fluctuations and turbulent boundary-layer separation. Arising in propelling nozzles or in air inlets, SBLI can cause engine disturbances and even engine failure. Over an aircraft wing surface,

Sebastian Arvidson

Saab Aeronautics, e-mail: sebastian.arvidson@saabgroup.com

Dept. of Applied Mechanics, Chalmers

Shia-Hui Peng

Swedish Defence Research Agency (FOI), e-mail: peng@foi.se

Dept of Applied Mechanics, Chalmers.

Lars Davidson

Depart. of Applied Mechanics, Chalmers, e-mail: lada@chalmers.se 
SBLI can deteriorate the aerodynamic performance and increase structural loads that may consequently lead to a decreased life-cycle time.

In numerical investigations on SBLI using CFD techniques, it is recognized that turbulence modeling remains a challenging aspect in order to accurately capture the flow physics prior to and after the SBLI-induced flow separation. Occurring at high speeds and large Reynolds numbers, SBLI triggers usually unsteady flow phenomena characterized by turbulent boundary-layer separation. For external flows (e.g. over a wing surface), the resulting flow separation may subsequently give rise of vortex motions. For internal flows (e.g. in a duct), SBLI may exaggerate the corner separation bubble.

In the present work, we examine the hybrid RANS-LES modeling approach in numerical simulations of SBLI-induced flow separation arising in a duct $[1,2,3]$ using the SA-DES [10], SA-DDES [9] and the algebraic HYB0 [6] models.

\section{SBLI flow configuration}

The computational configuration is illustrated in Figure 1 (a), also shown in Figure 1 (b) are the two grids (coarse and refined, cutting in a YZ-plan) used in the present work. The grids are of hexahedral type with about 3.2 and 7.6 million nodes respectively. Both grids have a refined region in the streamwise $(x-)$ direction to resolve the shock wave. For both grids, the first wall-normal grid node is generally located at $y^{+}<2$. Compared to the coarse grid, the refined grid has a doubled number of nodes in both directions normal to the duct walls in the bulk region.

The flow in the rectangular duct is accelerated over a convergent-divergent section reaching $M_{\infty}=1.4$. A shock wave is formed downstream, which interacts with the boundary layer and triggers flow separation in the duct corners.

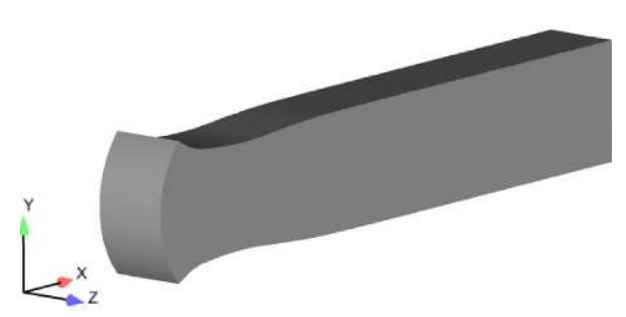

(a)

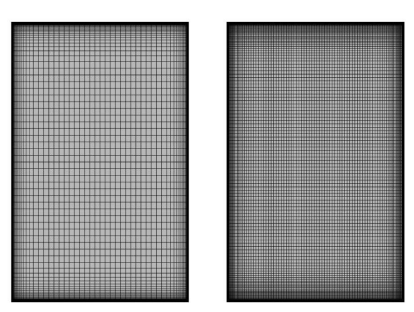

(b)

Fig. 1 (a) Computational configuration. (b) Grid resolution in a YZ-plan (left: coarse grid; right: refined grid).

In the experiment, a distinct symmetric shock wave is observed with its $\lambda$-type foot connecting with the near-wall boundary layers, see Figure 2 . The shock char- 
acteristics are strongly coupled with the formation of corner bubbles. In numerical simulations, modeling this coupling is essential to reach a correct prediction of SBLI. In the performed hybrid computations it has been observed that an increased strength of the $\lambda$-foot intensifies the growth of the flow separation downstream of the shock. An exaggerated corner bubble can, on the other hand, lead to a breakdown of the shock root resulting in a series of weak shocks with an asymmetric distribution in the duct.

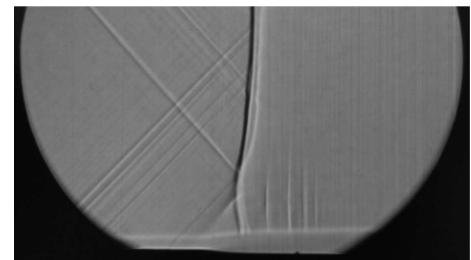

(a) Schlieren photo of shock wave with $\lambda$-foot.

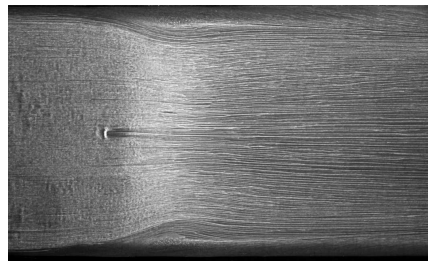

(b) Oil-flow visualization at bottom wall highlighting the corner bubbles.

Fig. 2 Experimental flow field.

The duct configuration used in the present computations has a length of $989 \mathrm{~mm}$ and a cross-section with dimensions of $178 \mathrm{~mm}$ in height and $114 \mathrm{~mm}$ in width. The shock wave in the experiment was measured at a location of $659 \mathrm{~mm}$ downstream the inlet. The observed recirculation bubbles in the duct corners are symmetric about the central section of the duct, having a width of approximately 10 percent of the duct width and a streamwise extension of about five times of its width, see Figure 2 (b). Hereafter the shock position will be referred to as $x=0$. Measured velocity data are available at $x=-30,0$ and $30 \mathrm{~mm}$ from the measured shock location in the central section over the bottom wall.

It is noted that the incoming boundary layer to the shock plays an important role in the prediction of the downstream SBLI properties. In all present turbulenceresolving simulations, the turbulent diffusion is often under-predicted in the boundary layer resulting in a too thick incoming boundary layer to the shock. The lower momentum near the wall makes the $\lambda$-foot weak and, consequently, an early corner flow separation is induced. Provided that the separation bubble is further enlarged, the $\lambda$-foot of the shock wave may collapse and leading to a breakdown of the standing shock wave.

\section{Simulation and modeling methods}

The computations have been conducted using an unstructured Navier-Stokes solver, Edge [4]. The turbulence-resolving simulations have been made using the SADES, its DDES variant and the algebraic HYB0 model. The low-Re-number $k-\omega$ 
model [7] (hereafter PDH LRN) has been included as a reference model through out this paper due to its good agreement with experimental data for this case. For steady state problems, Edge uses an explicit 3-stage Runge-Kutta scheme with the aid of an agglomerated multi-grid and residual smoothing for convergence acceleration. For unsteady simulations, a dual time-stepping approach is applied, combining the Runge-Kutta method with an implicit second-order scheme for physical time advancement. A second-order central differencing scheme has been used for spatial discretization.

In order to explore the time step effect, $\Delta t=4.65 \cdot 10^{-5}, 4.65 \cdot 10^{-6}$ and 4.65 . $10^{-7} \mathrm{~s}$ have been used with the HYB0 model. $\Delta t=4.65 \cdot 10^{-6} \mathrm{~s}$ was chosen for the grid and model comparison. The HYB0 model was used to explore the grid refinement effects. The information about turbulence models, computational grids and time steps are summarized in Table 1. Eight cases have been considered, of which six are simulations using hybrid RANS-LES methods and two are RANS computations taken as references for comparison.

Table 1 Summary of simulations

\begin{tabular}{llllll}
\hline Case & Mesh & Turb. model & $\Delta t[s]$ & $P_{\text {out }}[\mathrm{Pa}]$ & $\Delta x_{\text {shock }}[\mathrm{mm}]^{*}$ \\
\hline 1 & Coarse & HYB0 & $4.65 \cdot 10^{-5}$ & 82000 & 0 \\
2 & Coarse & HYB0 & $4.65 \cdot 10^{-6}$ & 82000 & +38 \\
3 & Coarse & SA-DES & $4.65 \cdot 10^{-6}$ & 82000 & +45 \\
4 & Coarse & SA-DDES & $4.65 \cdot 10^{-6}$ & 82000 & Collapsed shock \\
5 & Coarse & HYB0 & $4.65 \cdot 10^{-7}$ & 82000 & +35 \\
6 & Refined & HYB0 & $4.65 \cdot 10^{-6}$ & 82000 & +56 \\
7 & Coarse & PDH LRN $k-\omega$ & Steady state & 86200 & +2 \\
8 & Coarse & EARSM & Steady state & 87340 & 0 \\
\hline \multirow{2}{*}{ *Distance between simulated shock location and experimental location. $\Delta x_{\text {shock }}=x_{\text {sim. }}-x_{\text {exp }}}$.
\end{tabular}

On the inlet boundary the flow direction as well as the total pressure and temperature were specified according to the experimental data [1, 2], using $P_{0}=147.5 \mathrm{kPa}$ and $T_{0}=293 \mathrm{~K}$. Since the turbulence level was not measured at the inlet section in the experiment, the inflow turbulence intensity was set to $1 \%$ in all simulations.

The outlet pressure, to which the shock location is closely associated with the given inlet conditions, was not measured. In numerical simulations, it is noted that the predicted shock location depends also on the turbulence model used. To get the shock wave at the experimentally measured position, the outlet pressure has to be adjusted for different turbulence models. The outlet pressure adjustment was done in all RANS simulations. For the hybrid RANS-LES simulations presented in this paper, however, the outlet pressure was adjusted only for the simulation using the HYB0 model with a time step of $\Delta t=4.65 \cdot 10^{-5} \mathrm{~s}$ to match the experimental shock position. For the other hybrid RANS-LES simulations, this outlet pressure, $P_{\text {out }}=82 \mathrm{kPa}$, was used. As a result, the computed shock position from these simulations deviate from the experimental position. With the PDH LRN model, in RANS 
computations the effect on SBLI prediction has been investigated by changing the back pressure so that the shock location is shifted in the range of what has been predicted with the present hybrid RANS-LES simulations. It was found that the difference in the prediction of the corner bubble size and velocity profiles upstream the shock wave is negligibly small between the PDH LRN simulations. Thus, the shift in shock location in the hybrid simulations should not have any significant effect on the SBLI modeling.

To set the shock location consistent to the experimental measurement, a number of computations would be required in order to tune the back pressure. While this can be readily done in steady RANS computations, it is obviously very time-consuming in hybrid RANS-LES computations. Moreover, it is noted that, in spite of different shock locations predicted with the same back pressure, the boundary layer at 400 $\mathrm{mm}$ downstream from the inlet is almost identical in all computations. This suggests that, after accelerating, the incoming flow condition is the same when starting to approach the SBLI zone in all computations. By ruling out the effect of the back pressure, the differences in the predicted SBLI properties are thus essentially entailed by the modeling and by the temporal/spatial resolutions used. Provided that the specified back pressure has ensured that the Mach number reaches $M_{\infty}=1.4$ at $x=-30 \mathrm{~mm}$ upstream of the shock wave as indicated by the experiment, it is observed that, with the shock wave settled down at different locations within a certain distance (from the measured location), the predicted SBLI holds nearly identical flow properties but only a shift referring to the predicted shock location. For good resolution, moreover, it is desired that the predicted SBLI should take place within the region where the grid is refined. For appropriate comparisons with the experimental data at the same distance from the shock, the results have thus been extracted at a distance relative to the computed shock location.

All hybrid RANS-LES simulations were started from the RANS solution based on the PDH LRN $k-\omega$ model. Typically, ten times of convective time unit (CTU, namely, the time for a fluid particle passing through the duct at bulk flow speed) were needed to reach a fully developed flow after which the statistical analysis was conducted. Further, another ten CTUs were needed to get the resolved turbulence statistics and the mean flow field.

\subsection{Highlight of RANS-computed flow features}

Initially the described configuration was analyzed using RANS models. It was shown that some commonly used models in aerodynamic applications, such as the Spalart-Allmaras (SA) one-equation model [8] and the Menter SST model [5], fail to capture the SBLI giving asymmetrical solutions, as shown in Figure 3.

It was found that the symmetric shock wave can be reasonably reproduced by the PDH LRN and the Wallin-Johansson EARSM $k-\omega$ model [11]. The PDH LRN model gives a slightly over-predicted width of the shock-induced corner bubbles, as compared to the experiment visualization. The EARSM has hardly pronounced the 


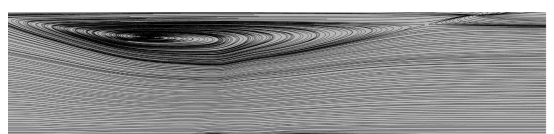

(a) Spalart-Allmaras

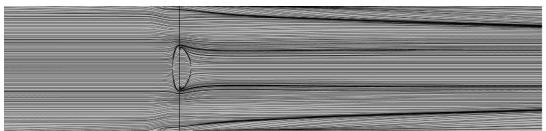

(c) EARSM

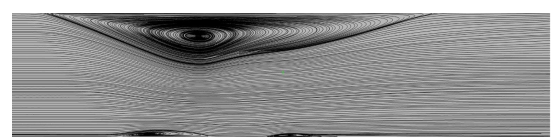

(b) Menter SST $k-\omega$

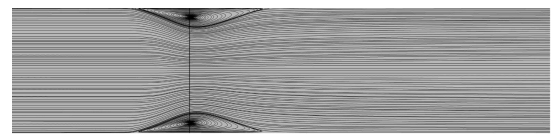

(d) PDH LRN $k-\omega$

Fig. 3 Skin friction pattern on bottom wall around the shock computed by different RANS models.

corner flow separation bubble but an incipient tendency towards boundary-layer separation. In addition, this model has captured a small separation bubble beneath the foot of the shock wave on the bottom wall surface, which is however not identified in experiment visualization.

In order to investigate the effect of the incoming boundary layer on the formation of the shock and on the shock-induced flow separation in the duct corners, a laminar boundary layer was specified from the inlet to the wall-parallel section covering the convergent-divergent passage. Little effect was observed on the RANS computations using either the PDH LRN model or the EARSM. Nonetheless, the corner separation bubble predicted by the PDH LRN $k-\omega$ model was slightly suppressed as desired.

The failure of the SA and the SST model in capturing the shock wave, and even the inaccurate prediction of the EARSM on the corner separation bubble, is phenomenologically related to the prediction of the separation onset and thereafter the extension of the separation bubble, as shown in Figure 3. Since the corner separation bubble is triggered in the presence of the shock wave, the representation of SBLI flow properties in the modeling should be of a significant issue for improving the flow prediction considered. The EARSM has predicted only a tendency of a much delayed separation onset at a location very close to the standing shock wave. The SST and SA model, on the other hand, have pronounced a far early onset of corner flow separation on one side of the duct, which has been extended undesirably in the streamwise direction and toward the opposite wall, where the separation bubble is largely suppressed. Note that the presence of the corner bubble plays a role of blockage in the duct, which may accelerate/decelerate the duct flow out of the corner bubbles. The over-predicted corner bubbles tend to make the shock wave reset in somewhat unsteady forms. In this case, a steady computation may lead to an asymmetric solution due to largely over-predicted corner bubbles that have induced unphysical unsteadiness in the SBLI flow properties. 


\section{Results and discussion}

All the hybrid RANS-LES computations are capable of resolving, to different extents, turbulent structures on the two meshes used. Most of the resolved structures are located in the shock-induced flow separation region in the duct corner, as illustrated by the resolved instantaneous flow pattern on the bottom wall surface in Figure 4. All simulations have captured the corner flow separation in the form of a three-dimensional recirculating bubble with its "eye" shifting slightly from the wall surface. For different models, grid resolutions and time steps (not shown), however, variations can be observed in the time-averaged flow fields, most sensibly on the shape and extension of the separation bubble. It is also observed that the size of the bubble is over-predicted in all simulations.

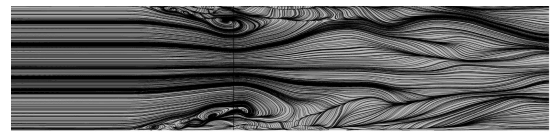

(a) Case 2: Instantaneous

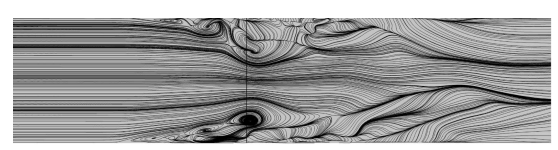

(c) Case 3: Instantaneous

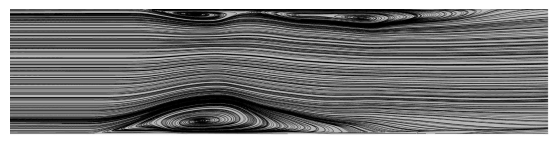

(e) Case 4: Instantaneous

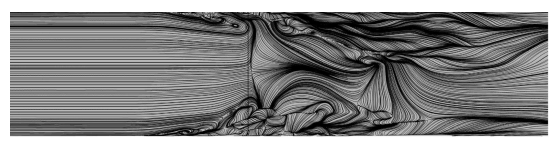

(g) Case 6: Instantaneous

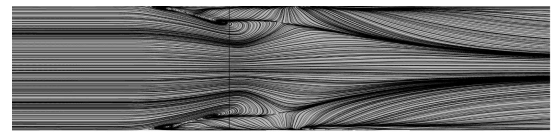

(b) Case 2: Time averaged

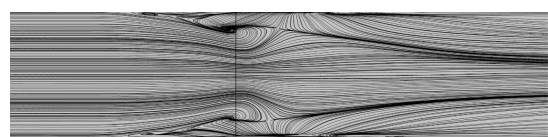

(d) Case 3: Time averaged

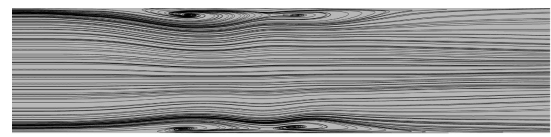

(f) Case 4: Time averaged

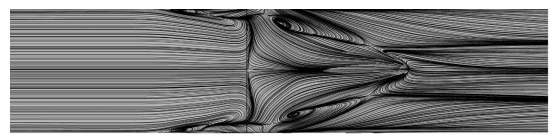

(h) Case 6: Time averaged

Fig. 4 Skin friction pattern on bottom wall around shock.

The resulting time-averaged flow fields with SA-DES and HYB0 are symmetric about the central section. The HYB0 (Case 2) and SA-DES (Case 3) computations have produced rather similar flow scenarios on the coarse grid with the same time step. For the SA-DES and HYB0 simulations, a major part of the corner bubble is treated in LES mode. The shielding function used in DDES does not respond properly to the separated flow in the corner bubble, where only very large turbulent structures are resolved, since a large part of the separation is modeled by the RANS mode. For the SA-DES and HYB0 simulations, a separation bubble can clearly be identified in each corner with resolved turbulent structures enclosed. With SA- 
DDES, on the other hand, the instantaneous corner separation bubble moves from side to side, bursting and rebuilding with a low frequency. Due to this low frequency behaviour, the corner separation bubbles in the SA-DDES simulation require a much longer simulation time for time-averaging analysis. Since ten CTUs have been used for time averaging in all simulations, this might explain why a slightly asymmetric time-averaged flow field is achieved with SA-DDES as seen in Figure 4 (f).

On the refined grid, only one simulation with the HYB0 model (Case 6) is available so far. A relatively rich set of hairpin-type vortices are observed being detached from the wall surface and evolving in the corner separation region, as shown in Figure 5. The shock predicted in this simulation tends to initiate a flow separation over the whole wall surface, and the flow separation is further enlarged downstream forming a large recirculation bubble which is oriented towards the duct center as illustrated in Figures $4(\mathrm{~g})$ and $(\mathrm{h})$.

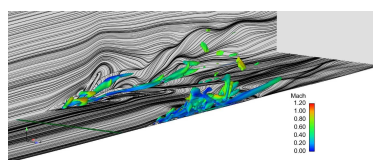

(a) Case 2

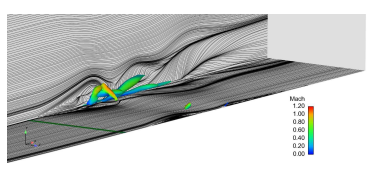

(c) Case 4

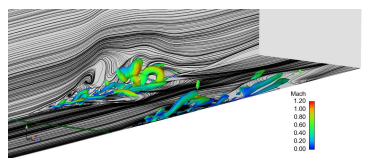

(b) Case 3

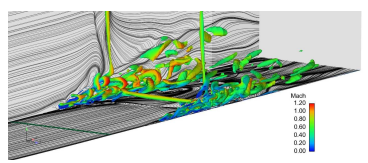

(d) Case 6

Fig. 5 Q-criterion colored by Mach number in the lower duct corners.

It was found that, a time step of $\Delta t=4.65 \cdot 10^{-6} \mathrm{~s}$, which corresponds to an acoustic CFL number of 1 in the bulk flow at the shock, is required for the used grids in order to capture the fluctuations in the SBLI zone. $\Delta t=4.65 \cdot 10^{-5} \mathrm{~s}$ makes the shock breakdown even more severe, while $\Delta t=4.65 \cdot 10^{-7} \mathrm{~s}$ does not contribute to much improvement in the prediction of shock intensity and velocity profiles in the shock region, as shown in Figure 6 and 7 respectively. However, with $\Delta t=$ $4.65 \cdot 10^{-7} \mathrm{~s}$ the growth of the time-averaged corner separation bubble downstream $x=30 \mathrm{~mm}$ is even more enlarged than with $\Delta t=4.65 \cdot 10^{-6} \mathrm{~s}$.

The pressure distributions along the centerlines of the bottom wall surface and the duct are presented in Figure 6 (a) and (b), respectively. Note that the shock location is referred to $x=0$. Obviously, all the unsteady simulations have under-estimated the strength of the shock wave, for which the pressure gradient over the shock location has been predicted much less steep over the wall surface compared to the experiment. Over the central section of the duct, as shown in Figure 6 (b), the predicted pressure gradient over the shock location is sharp with a relatively mild increase towards the shock front as compared to the RANS prediction. The hybrid RANS-LES computations, moreover, have yielded an additional re-compression shock after an 
immediate accelerating expansion downstream of the main shock. This unphysical property has been caused by the over-predicted off-wall extension of the corner bubbles, which push the incoming air towards the duct center and accelerate the flow there. Downstream of the "bubble eye" and away from the recirculation bubble, the flow-through part forms a divergent section in the duct, which has facilitated the form of the unphysical re-compression shock.

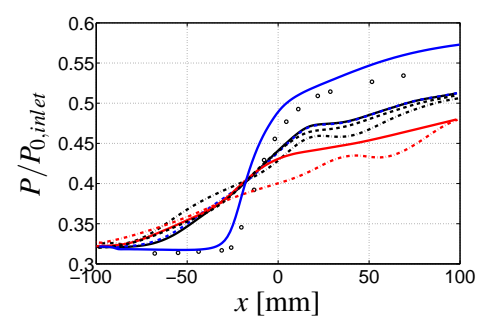

(a) Bottom wall.

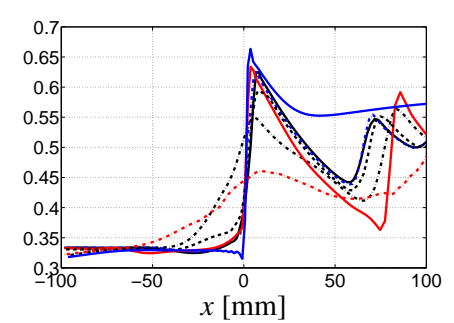

(b) Symmetry axis at $y / H=0.5$.

Fig. 6 Static pressure distributions across the shock, $H$ is duct height. ०: Experiment; _. - : Case 1;

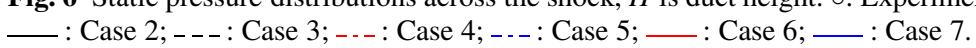

The over-predicted size of the corner separation bubble may have stemmed in part from the inaccurate prediction of the boundary layer approaching the shock. Figure 7 (a)-(c) presents the velocity profiles in the boundary layer over the bottom wall on the symmetric section. All the simulations have produced an identical velocity profile in the boundary layer at $400 \mathrm{~mm}$ downstream of the inlet. Moving towards the shock location at $x=-100 \mathrm{~mm}$, variations are found in the velocity profiles due to different models and grid resolutions while the time step has negligible effects upstream the shock. Unlike the PDH LRN computation, the boundary layer is inaccurately predicted in all hybrid simulations at $x=-30 \mathrm{~mm}$. At the shock location $(x=0)$, the predicted velocity profiles agree reasonably well with the experimental data, but the SA-DDES computation has over-predicted the near-wall velocity since no distinct shock is produced due to the elongated corner separation bubble shown in Figure 4 (f) which is also clearly reflected in the pressure distributions in Figure 6 . The deficit in the velocity profile of the boundary layer at $x=-30 \mathrm{~mm}$ is reflected by the density. Although not shown here, it was found that the more the deficit is in the velocity of the boundary layer, the larger the predicted density became. In future work, the compressibility effect on the modeling should be further explored.

With HYB0 on the refined grid, the prediction of the boundary layer is even worse than on the coarse grid. This is related to the resolved boundary-layer separation, see Figure $4(\mathrm{~g})$ and $(\mathrm{h})$. Figure 7 (d) shows an estimation of where the RANS-LES interface is located for SA-DDES and SA-DES on the coarse grid and HYB0 on both the coarse and refined grids. It is shown that HYB0 on the refined grid and SA-DES on the coarse grid switches from RANS to LES inside the boundary layer from $x=-100 \mathrm{~mm}$ and all the way downstream through the SBLI zone. Note that HYB0 is similar to a wall-modeled LES approach, which should be able 


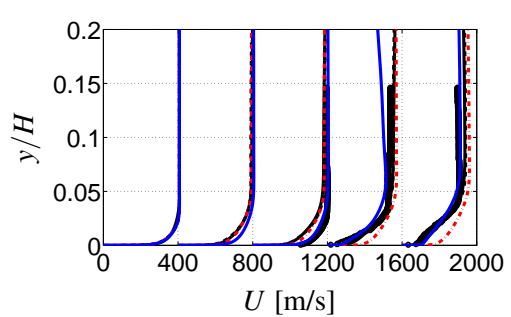

(a) Effect of model.

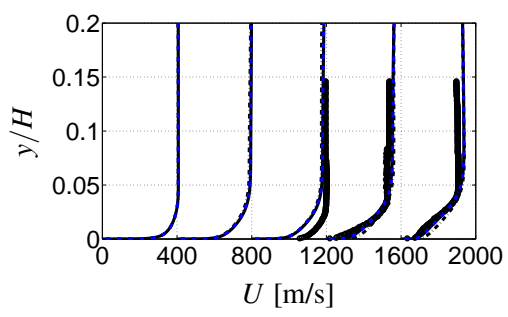

(c) Effect of time step.

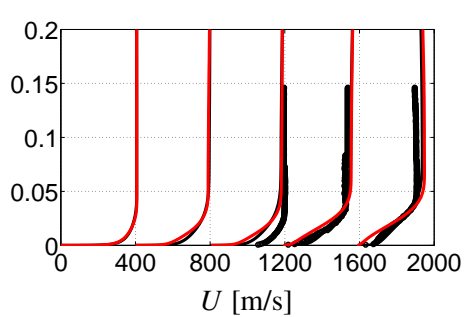

(b) Effect of mesh.

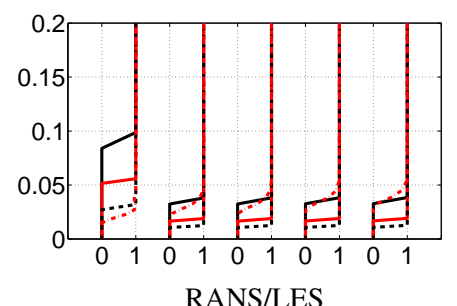

(d) RANS-LES interface.

Fig. 7 Velocity profiles and RANS-LES switch location on the symmetric central section at 400 $\mathrm{mm}$ downstream the inlet (the first profile on the left-hand side in each figure) and at $x=-100,-30$, $0,30 \mathrm{~mm}$ from the shock, $H$ is duct height. (a) Coarse grid, $\Delta t=4.65 \cdot 10^{-6} s$. (b) HYB0 model, $\Delta t=4.65 \cdot 10^{-6} s$. (c) HYB0 model, coarse grid. (d) RANS-LES switch location, RANS: 0 and

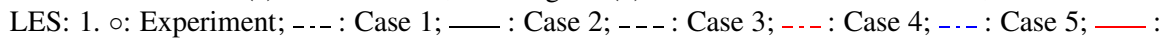
Case $6 ;-$ : Case 7

to handle the switch from RANS to LES inside the boundary layer provided that the grid is sufficiently fine. However, the grid upstream the SBLI zone where high velocities are present have shown to be too coarse even in the refined case for a model such as HYB0. This contributes to an inaccurate prediction of the boundarylayer flow due to an under-resolved LES, which in turn contributes to the increased over-prediction of the corner bubble together with the stronger shock for this case. Downstream of the shock at $x=30 \mathrm{~mm}$, the HYB0 simulation on the refined grid gives a different shape compared to the measured profile in association to the overpredicted separation bubble.

\section{Concluding remarks}

Testing of hybrid RANS-LES modeling has been conducted in comparison with available experimental data for the SBLI taking place in a rectangular duct flow at $M_{\infty}=1.4$. All the studied hybrid RANS-LES models have failed to capture the underlying physics of the shock-induced corner flow separation. The standing shock wave is collapsed in association to the prediction of the corner flow separation. 
Contrary to flows past a bluff-body with massive separation, the shock-induced flow separation is of shallow type and is enclosed in the duct corner and partly embedded in the boundary layer. This means that the separation bubble and the shock wave standing through the near-wall boundary layer have been dealt with by both the LES and RANS modes. This is very challenging for hybrid RANS-LES modeling, particularly for the RANS-LES interface.

The modeling demands special attention paid to the RANS-LES interface in relation to the local grid resolution. Even for DDES-type models, the use of a shielding function is not justified in the region of the separation zone. The shielding function has played a role as desired in the boundary layer upstream the SBLI zone but does not respond properly to the separated region which, as with the SST model, has predicted a much earlier onset of the corner flow separation and has made the corner separation bubble largely over-predicted. The poorly predicted shock wave and corner separation bubble in the SA-DDES simulation is not only due to the DDES formulation but also to the underlying SA RANS model incorporated. The location of the simulated bubble onset is thus very important and essential in the prediction of corner bubble size. A too early predicted onset and an exaggerated corner bubble will breakdown the shock wave and form an unphysical re-compression shock.

To capture the flow physics and resolve the corner separation bubbles a wallmodeled LES approach, like the HYB0 model, might be an alternative provided that the grid is sufficiently fine in the LES region. The grids used in this study have shown to be too coarse, at least upstream the SBLI zone, and hence not fulfill the requirement for such a model. The use of a wall-modeled LES approach in the full domain for internal flows involving SBLI is computationally very costly and is today impossible to handle in industrial applications.

The study of the current SBLI flow has shown that a time step corresponding to an acoustic CFL number of 1 is required in the bulk flow at the shock for the grids used in order to capture the fluctuations in the SBLI zone. Using the refined grid, large differences in the prediction of the corner separation and shock intensity were found, as compared to the coarse grid.

In future work the modeling issues will be considered including the compressibility effect. Especially the work will focusing on further investigating the DDES approach. To adapt it for shallow separations and incorporating a RANS base model which has the capability to predict internal SBLI flows. A zonal formulation is interesting and will be studied. Such a formulation will treat the region upstream the SBLI zone with a RANS-like mesh and incorporate a URANS model which can produce a boundary layer in good agreement with experimental data. The SBLI zone will use a fine grid and a LES based approach to accurately resolve the turbulent structures enclosed in the corner separation bubbles.

Acknowledgements This work was primarily founded by the Swedish National Flight Research Program (NFFP) and Saab Aeronautics, and was partially conducted within the EU project ATAAC, Contract No. 233710. Computational resources was supported by the Swedish National Infrastructure for Computing (SNIC). 


\section{References}

1. Bruce, P.J.K., Babinsky, H.: Unsteady shock wave dynamics. Journal of Fluid Mechanics $\mathbf{6 0 3}$, 463-473 (2008)

2. Bruce, P.J.K., Babinsky, H., Tartinville, B., Hirch, C.: An experimental and numerical study of an oscillating transonic shock wave in a duct. In: 48th AIAA Aerospace Science Meeting. Orlando, 2010-925 (2010)

3. Doerffer, P.: Description of Test Section and Measurements in a Nozzle for Shock Upstream Mach Number M=1.45. In: Peng, S-H., Doerffer, P., Haase, W., (eds.) Progress in Hybrid RANS-LES Modelling, pp. 339-344. Springer, Heidelberg (2010)

4. Eliasson, P.: EDGE, a Navier-Stokes Solver for Unstructured Grids. Scientific report, FOI-R0298-SE, Computational Aerodynamics Department, Aeronautics Division, FOI (2001)

5. Menter, F.R.: Two-Equation Eddy-Viscosity Turbulence Models for Engineering Applications. AIAA Journal 32, 1598-1605 (1994)

6. Peng, S-H.: Hybrid RANS-LES Modeling Based on Zero- and One-Equation Models For Turbulent Flow Simulation. In: Proceedings of the 4th International Symposium on Turbulence and Shear Flow Phenomena. Williamsburg (2005)

7. Peng, S-H., Davidson, L., Holmberg, S.: A Modified Low-Reynolds-Number $k-\omega$ Model for Recirculating Flows. Journal of Fluids Engineering 119, 867-875 (1997)

8. Spalart, P.R., Allmaras, S.R.: A One-Equation Turbulence Model for Aerodynamic Flows. La Recherche Aerospatiale 1, 5-21 (1994)

9. Spalart, P.R., Deck, S., Shur, M.L., Squires, K.D.: A new version of detached-eddy simulation, resistant to ambiguous grid densities. Theory of Computational Fluid Dynamics 20, pp. 181-195 (2006)

10. Spalart, P.R., Jou, W-H., Strelets, M., Allmaras, R.: Comments on the Feasability of LES for Wings, and on a Hybrid RANS/LES Approach. In: Advances in DNS/LES, pp. 137-147. Ruston, Lousiana (1997)

11. Wallin, S., Johansson, A.: An explicit algebraic Reynolds stress model for incompressible and compressible turbulent flows. Journal of Fluid Mechanics 403, 89-132 (2000) 